\title{
A sum analogous to the high-dimensional Kloosterman sums and its upper bound estimate
}

Yijun Li $i^{1,2}$ and Di Han ${ }^{1 *}$

"Correspondence: handi515@.com 'Department of Mathematics, Northwest University, Xi'an, Shaanxi, P.R. China

Full list of author information is available at the end of the article

\begin{abstract}
The main purpose of this paper is, using the properties of Gauss sums and the estimate for the generalized exponential sums, to study the upper bound estimate problem of one kind sums analogous to the high-dimensional Kloosterman sums and to give some interesting mean value formula and an upper bound estimate for it.

MSC: 11L05

Keywords: a sum analogous to the high-dimensional Kloosterman sums; Gauss sums; upper bound estimate; mean value
\end{abstract}

\section{Introduction}

For any integer $q \geq 3$, the high-dimensional Kloosterman sums $K\left(c_{1}, c_{2}, \ldots, c_{k}, m ; q\right)$ are defined as follows:

$$
K\left(c_{1}, c_{2}, \ldots, c_{k}, m ; q\right)=\sum_{a_{1}=1}^{q} \cdots \sum_{a_{k}=1}^{q} e\left(\frac{c_{1} a_{1}+\cdots+c_{k} a_{k}+m \bar{a}_{1} \cdots \bar{a}_{k}}{q}\right),
$$

where $e(x)=e^{2 \pi i x}, \sum_{a_{i}=1}^{\prime q}$ denotes the summation over all integers $1 \leq a_{i} \leq q$ such that $\left(a_{i}, q\right)=1, c_{i}$ and $m$ are integers with $(m, q)=1, \bar{a}_{i}$ denotes the solution of the congruent equation $x \cdot a_{i} \equiv 1 \bmod q(i=1,2, \ldots, k)$.

There are several results on the properties of the Kloosterman sums $K\left(c_{1}, c_{2}, \ldots, c_{k}, m ; q\right)$. For example, see [1, 2] and [3]. Related works can also be found in [4-8] and [9].

In this paper, we consider a sum analogous to the high-dimensional Kloosterman sums as follows:

$$
S\left(c_{1}, c_{2}, \ldots, c_{k}, m, \chi ; q\right)=\sum_{a_{1}=1}^{q} \cdots \sum_{a_{k}=1}^{q} \chi\left(c_{1} a_{1}+\cdots+c_{k} a_{k}+m \bar{a}_{1} \cdots \bar{a}_{k}\right),
$$

where $\chi$ is a Dirichlet character $\bmod q$.

If $k=1$ and $q=p$ (an odd prime), then for any integer $a$ with $(a, p)=1$, applying the Fermat little theorem, one can deduce $a^{p-2} \equiv \bar{a} \bmod p$. So, the sum (1.1) becomes

$$
\sum_{a=1}^{p-1} \chi(c a+m \bar{a})
$$

๑) 2013 Li and Han; licensee Springer. This is an Open Access article distributed under the terms of the Creative Commons Attribution License (http://creativecommons.org/licenses/by/2.0), which permits unrestricted use, distribution, and reproduction in any medium, provided the original work is properly cited. 
It is a special case of the general polynomial character sums

$$
\sum_{a=N+1}^{N+M} \chi(f(a))
$$

where $M$ and $N$ are any positive integers and $f(x)$ is a polynomial. Let $\chi$ be a $q$ th-order character $\bmod p$. If $f(x)$ is not a perfect $q$ th power $\bmod p$, then from Weil's classical work (see [10]), we can deduce the estimate

$$
\sum_{x=N+1}^{N+M} \chi(f(x)) \ll p^{\frac{1}{2}} \ln p
$$

where ' «' constant depends only on the degree of $f(x)$. Some related results can also be found in [11-13] and [14].

Now we are concerned with the upper bound estimate problem of (1.1). Regarding this contents, it seems that nobody has yet studied it, at least we have not seen any related result before. The problem is interesting because it can reflect some new properties of character sums. The main purpose of this paper is, using the analytic methods and the properties of Gauss sums, to study this problem and give a sharp upper bound estimate for (1.1). That is, we prove the following conclusions.

Theorem 1 Let $p$ be an odd prime, let $k$ be a positive integer with $k \geq 2$, and let $\chi$ be any non-principal character modp. Then for any integers $c_{1}, c_{2}, \ldots, c_{k}$ and $m$ with $\left(c_{1} c_{2} \cdots c_{k} m, p\right)=1$, we have the identity

$$
\left|S\left(c_{1}, c_{2}, \ldots, c_{k}, m, \chi ; p\right)\right|= \begin{cases}p^{\frac{k}{2}} & \text { if }(k+1, p-1)=1, \\ 0 & \text { if }(k+1) \mid(p-1) \text { and } \chi^{\frac{p-1}{k+1}} \neq \chi_{0},\end{cases}
$$

where $\chi_{0}$ denotes the principal character $\bmod p$.

Theorem 2 Let $p$ be an odd prime, let $k$ be a positive integer with $k \geq 2$, and let $\chi$ be any non-principal character $\bmod p$. Then for any integers $c_{1}, c_{2}, \ldots, c_{k}$ and $m$ with $\left(c_{1} c_{2} \cdots c_{k} m, p\right)=1$, we have the estimate

$$
\left|S\left(c_{1}, c_{2}, \ldots, c_{k}, m, \chi ; p\right)\right| \leq(k+1) \cdot p^{\frac{k}{2}}
$$

Theorem 3 Let $p$ and $q$ be two odd primes, let $r$ be any qth non-residue $\bmod p$. Then for any integers $c_{1}, c_{2}, \ldots, c_{q-1}$ with $\left(c_{1} c_{2} \cdots c_{q-1}, p\right)=1$, we have the identity

$$
\begin{aligned}
& \sum_{i=0}^{q-1}\left[\sum_{a_{1}=1}^{p-1} \sum_{a_{2}=1}^{p-1} \cdots \sum_{a_{q-1}=1}^{p-1}\left(\frac{c_{1} a_{1}+c_{2} a_{2}+\cdots+c_{q-1} a_{q-1}+r^{i} \bar{a}_{1} \bar{a}_{2} \cdots \bar{a}_{q-1}}{p}\right)\right]^{2} \\
& \quad= \begin{cases}q^{2} \cdot p^{q-1} & \text { if } q \mid(p-1), \\
q \cdot p^{q-1} & \text { if }(q, p-1)=1 .\end{cases}
\end{aligned}
$$

If $p \equiv 1 \bmod 4$, then the above formula also holds for $q=2$, where $\left(\frac{*}{p}\right)$ denotes the Legendre symbol. 
Taking $p=4 m+1$ and $q=2$ in Theorem 3 , note that $2 \mid(p-1)$, we may immediately deduce the following.

Corollary Let $p$ be an odd prime with $p \equiv 1 \bmod 4$, then we have the identity

$$
p=\left(\sum_{a=1}^{\frac{p-1}{2}}\left(\frac{a+r \bar{a}}{p}\right)\right)^{2}+\left(\sum_{b=1}^{\frac{p-1}{2}}\left(\frac{b+s \bar{b}}{p}\right)\right)^{2}
$$

where $r$ and s are any two integers such that $\left(\frac{r}{p}\right) \cdot\left(\frac{s}{p}\right)=-1$.

This gives another proof for a classical work in elementary number theory (i.e., see [15] Theorems 4-11): For any prime $p$ with $p \equiv 1 \bmod 4$, there exist two positive integers $x$ and $y$ such that $p=x^{2}+y^{2}$.

\section{Several lemmas}

To complete the proof of our theorems, we need the following basic lemmas.

Lemma 1 Let $p$ be an odd prime, let $\chi$ be any non-principal character $\bmod p$, and let $k$ be any positive integer such that $(k, p-1)=1$ or $k \mid p-1$. Then for any integer $m$ with $(m, p)=1$, we have the identity

$$
\sum_{a=1}^{p-1} \chi(a) e\left(\frac{m a^{k}}{p}\right)= \begin{cases}\bar{\chi}^{r}(m) \cdot \tau\left(\chi^{r}\right) & \text { if }(k, p-1)=1, \\ 0 & \text { if } k \mid(p-1) \text { and } \chi^{\frac{p-1}{k}} \neq \chi_{0}, \\ \bar{\chi}_{1}(m) \cdot \sum_{i=0}^{k-1} \bar{\chi}_{k}^{i}(m) \tau\left(\chi_{1} \chi_{k}^{i}\right) & \text { if } k \mid(p-1) \text { and } \chi^{\frac{p-1}{k}}=\chi_{0},\end{cases}
$$

where $r \cdot k \equiv 1 \bmod (p-1), \chi_{0}$ denotes the principal character $\bmod p, \chi_{k}$ denotes any $k$-order character $\bmod p$ and $\chi_{1}^{k}=\chi$.

Proof If $(k, p-1)=1$, then there exists one integer $r$ with $(r, p-1)=1$ such that $r \cdot k \equiv$ $1 \bmod (p-1)$. This time, for any integer $a$ with $(a, p)=1$, we have $a^{r k} \equiv a \bmod p$. If $a$ passes through a reduced residue system $\bmod p$, then $a^{r}$ also passes through a reduced residue system $\bmod p$. Therefore, we have

$$
\begin{aligned}
\sum_{a=1}^{p-1} \chi(a) e\left(\frac{m a^{k}}{p}\right) & =\sum_{a=1}^{p-1} \chi\left(a^{r}\right) e\left(\frac{m a^{r k}}{p}\right) \\
& =\sum_{a=1}^{p-1} \chi^{r}(a) e\left(\frac{m a}{p}\right)=\bar{\chi}^{r}(m) \cdot \tau\left(\chi^{r}\right) .
\end{aligned}
$$

If $k>1$ and $k \mid(p-1)$ with $\chi^{\frac{p-1}{k}} \neq \chi_{0}$, then there must exist an integer $n$ with $(n, p)=1$ such that $\chi^{\frac{p-1}{k}}(n) \neq 1$. For this $n$, we have

$$
\begin{aligned}
\sum_{a=1}^{p-1} \chi(a) e\left(\frac{m a^{k}}{p}\right) & =\sum_{a=1}^{p-1} \chi\left(a \cdot n^{\frac{p-1}{k}}\right) e\left(\frac{m\left(a \cdot n^{\frac{p-1}{k}}\right)^{k}}{p}\right) \\
& =\chi\left(n^{\frac{p-1}{k}}\right) \sum_{a=1}^{p-1} \chi(a) e\left(\frac{m a^{k} n^{p-1}}{p}\right)=\chi^{\frac{p-1}{k}}(n) \sum_{a=1}^{p-1} \chi(a) e\left(\frac{m a^{k}}{p}\right)
\end{aligned}
$$


or

$$
\left(1-\chi \frac{p-1}{k}(n)\right) \sum_{a=1}^{p-1} \chi(a) e\left(\frac{m a^{k}}{p}\right)=0
$$

Since $\chi^{\frac{p-1}{k}}(n) \neq 1$, from the above identity, we have

$$
\sum_{a=1}^{p-1} \chi(a) e\left(\frac{m a^{k}}{p}\right)=0
$$

If $\chi^{\frac{p-1}{k}}=\chi_{0}$, then $\chi$ must be a $k$ th character $\bmod p$, so there exists one character $\chi_{1} \bmod p$ such that $\chi=\chi_{1}^{k}$. Let $\chi_{k}$ be a $k$-order character $\bmod p\left(i . e\right.$., $\left.\chi_{k}^{k}=\chi_{0}\right)$, then for any integer $a$ with $(a, p)=1$, note that

$$
1+\chi_{k}(a)+\chi_{k}^{2}(a)+\cdots+\chi_{k}^{k-1}(a)= \begin{cases}k & \text { if } a \text { is a } k \text { th } \operatorname{residue~} \bmod p \\ 0, & \text { otherwise }\end{cases}
$$

From the properties of Gauss sums, we have

$$
\begin{aligned}
\sum_{a=1}^{p-1} \chi(a) e\left(\frac{m a^{k}}{p}\right) & =\sum_{a=1}^{p-1} \chi_{1}^{k}(a) e\left(\frac{m a^{k}}{p}\right)=\sum_{a=1}^{p-1} \chi_{1}\left(a^{k}\right) e\left(\frac{m a^{k}}{p}\right) \\
& =\sum_{a=1}^{p-1} \chi_{1}(a)\left(1+\chi_{k}(a)+\chi_{k}^{2}(a)+\cdots+\chi_{k}^{k-1}(a)\right) e\left(\frac{m a}{p}\right) \\
& =\bar{\chi}_{1}(m) \cdot \sum_{i=0}^{k-1} \bar{\chi}_{k}^{i}(m) \tau\left(\chi_{1} \chi_{k}^{i}\right) .
\end{aligned}
$$

Now Lemma 1 follows from (2.1), (2.2) and (2.3).

Lemma 2 Let $p$ and $q$ be two odd primes with $q \mid(p-1)$, and let $\chi_{q}$ be any $q$-order character $\bmod p$. Then for any integers $c_{1}, c_{2}, \ldots, c_{q-1}$ and $m$ with $\left(m c_{1} c_{2} \cdots c_{q-1}, p\right)=1$, we have the identities

(I) $\sum_{a_{1}=1}^{p-1} \sum_{a_{2}=1}^{p-1} \cdots \sum_{a_{q-1}=1}^{p-1}\left(\frac{c_{1} a_{1}+c_{2} a_{2}+\cdots+c_{q-1} a_{q-1}+m \bar{a}_{1} \bar{a}_{2} \cdots \bar{a}_{q-1}}{p}\right)$

$$
=\left(\frac{n}{p}\right) \cdot \tau^{q-1}\left(\chi_{2}\right)\left(\sum_{i=0}^{q-1} \chi_{q}^{i}(n) \frac{\tau^{q}\left(\chi_{2} \chi_{q}^{i}\right)}{\tau^{q}\left(\chi_{2}\right)}\right) ;
$$

(II) $\sum_{a=1}^{p-1}\left(\frac{a+m \bar{a}}{p}\right)= \begin{cases}\frac{\bar{\chi}_{1}(m)}{\tau\left(\chi_{2}\right)}\left(\tau^{2}\left(\chi_{1}\right)+\left(\frac{m}{p}\right) \tau^{2}\left(\bar{\chi}_{1}\right)\right) & \text { if } p \equiv 1 \bmod 4, \\ 0 & \text { if } p \equiv 3 \bmod 4,\end{cases}$

where $\left(\frac{*}{p}\right)=\chi_{2}$ denotes the Legendre symbol, $n=m c_{1} c_{2} \cdots c_{q-1}$ and $\chi_{1}^{2}=\chi_{2}$. 
Proof If $q$ is an odd prime, then $\chi_{2}^{q}=\chi_{2}$ and $\bar{\chi}_{2}=\chi_{2}$, so applying (2.3) and the properties of Gauss sums, we have

$$
\begin{aligned}
\sum_{a_{1}=1}^{p-1} & \sum_{a_{2}=1}^{p-1} \cdots \sum_{a_{q-1}=1}^{p-1}\left(\frac{c_{1} a_{1}+c_{2} a_{2}+\cdots+c_{q-1} a_{q-1}+m \bar{a}_{1} \bar{a}_{2} \cdots \bar{a}_{q-1}}{p}\right) \\
= & \sum_{a_{1}=1}^{p-1} \sum_{a_{2}=1}^{p-1} \cdots \sum_{a_{q-1}=1}^{p-1}\left(\frac{a_{1}+a_{2}+\cdots+a_{q-1}+n \bar{a}_{1} \bar{a}_{2} \cdots \bar{a}_{q-1}}{p}\right) \\
= & \frac{1}{\tau\left(\chi_{2}\right)} \sum_{a_{1}=1}^{p-1} \sum_{a_{2}=1}^{p-1} \cdots \sum_{a_{q-1}=1}^{p-1} \sum_{b=1}^{p-1} \chi_{2}(b) e\left(\frac{b\left(a_{1}+\cdots+a_{q-1}\right)+b n \bar{a}_{1} \cdots \bar{a}_{q-1}}{p}\right) \\
= & \frac{1}{\tau\left(\chi_{2}\right)} \sum_{a_{1}=1}^{p-1} \sum_{a_{2}=1}^{p-1} \cdots \sum_{a_{q-2}=1}^{p-1} \sum_{b=1}^{p-1} \chi(b) e\left(\frac{b\left(1+a_{1}+\cdots+a_{q-2}\right)}{p}\right) \\
= & \times \sum_{a_{q-1}=1}^{p-1} \chi_{2}\left(a_{q-1}\right) e\left(\frac{b n \bar{a}_{q-1}^{q} \bar{a}_{1} \cdots \bar{a}_{q-2}}{p}\right) \\
& \frac{1}{p\left(\chi_{2}\right)} \sum_{a_{1}=1}^{p-1} \sum_{a_{2}=1}^{p-1} \cdots \sum_{a_{q-2}=1}^{p-1} \sum_{b=1}^{p-1} \chi(b) e\left(\frac{b\left(1+a_{1}+\cdots+a_{q-2}\right)}{p}\right) \\
& \times \sum_{c=1}^{p-1} \chi_{2}(c)\left(1+\chi_{q}(c)+\cdots+\chi_{q}^{q-1}(c)\right) e\left(\frac{b n c \bar{a}_{1} \cdots \bar{a}_{q-2}}{p}\right) \\
= & \left(\frac{n}{p}\right) \cdot \tau^{q-1}\left(\chi_{2}\right)\left(\sum_{i=0}^{q-1} \chi_{q}^{i}(n) \frac{\tau^{q}\left(\chi_{2} \chi_{q}^{i}\right)}{\tau q\left(\chi_{2}\right)}\right) .
\end{aligned}
$$

This proves formula (I).

To prove formula (II), note that if $p \equiv 3 \bmod 4$, then $\chi_{2}$ must be an odd character $\bmod p$ (i.e., $\chi_{2}(-1)=-1$ ) so that

$$
\begin{aligned}
\sum_{a=1}^{p-1}\left(\frac{a+m \bar{a}}{p}\right) & =\sum_{a=1}^{p-1}\left(\frac{p-a+m \overline{p-a}}{p}\right) \\
& =\left(\frac{-1}{p}\right) \sum_{a=1}^{p-1}\left(\frac{a+m \bar{a}}{p}\right)=-\sum_{a=1}^{p-1}\left(\frac{a+m \bar{a}}{p}\right)
\end{aligned}
$$

or

$$
\sum_{a=1}^{p-1}\left(\frac{a+m \bar{a}}{p}\right)=0 .
$$

If $p \equiv 1 \bmod 4$, then there exists one character $\chi_{1} \bmod p$ such that $\chi_{1}^{2}=\chi_{2}$. Note that $\chi_{1}^{3}=$ $\bar{\chi}_{1}$ and $\chi_{2} \bar{\chi}_{1}=\chi_{1}$; from the properties of Gauss sums, we have

$$
\begin{aligned}
\sum_{a=1}^{p-1}\left(\frac{a+m \bar{a}}{p}\right) & =\frac{1}{\tau\left(\chi_{2}\right)} \sum_{a=1}^{p-1} \sum_{b=1}^{p-1} \chi_{2}(b) e\left(\frac{b(a+m \bar{a})}{p}\right) \\
& =\frac{1}{\tau\left(\chi_{2}\right)} \sum_{b=1}^{p-1} \chi_{2}(b) \sum_{a=1}^{p-1} \chi_{2}(\bar{a}) e\left(\frac{b+m b \bar{a}^{2}}{p}\right)
\end{aligned}
$$




$$
\begin{aligned}
& =\frac{1}{\tau\left(\chi_{2}\right)} \sum_{b=1}^{p-1} \chi_{2}(b) e\left(\frac{b}{p}\right) \sum_{a=1}^{p-1} \chi_{1}^{2}(a) e\left(\frac{m b a^{2}}{p}\right) \\
& =\frac{1}{\tau\left(\chi_{2}\right)} \sum_{b=1}^{p-1} \chi_{2}(b) e\left(\frac{b}{p}\right) \sum_{a=1}^{p-1} \chi_{1}(a)\left(1+\chi_{2}(a)\right) e\left(\frac{m b a}{p}\right) \\
& =\frac{\bar{\chi}_{1}(m)}{\tau\left(\chi_{2}\right)}\left(\tau^{2}\left(\chi_{1}\right)+\left(\frac{m}{p}\right) \tau^{2}\left(\bar{\chi}_{1}\right)\right) .
\end{aligned}
$$

This proves Lemma 2.

\section{Proof of the theorems}

In this section, we complete the proof of our theorems. First we prove Theorems 1 and 2. Let $n=m c_{1} c_{2} \cdots c_{k},(k+1, p-1)=d$. If $d=1$, we can assume $r(k+1) \equiv 1 \bmod (p-1)$, then from Lemma 1 , the properties of a reduced residue system $\bmod p$ and Gauss sums, we have

$$
\begin{aligned}
& S\left(c_{1}, c_{2}, \ldots, c_{k}, m, \chi ; p\right) \\
& =\sum_{a_{1}=1}^{p-1} \cdots \sum_{a_{k}=1}^{p-1} \chi\left(a_{1}+\cdots+a_{k}+m c_{1} c_{2} \cdots c_{k} \cdot \bar{a}_{1} \cdots \bar{a}_{k}\right) \\
& =\frac{1}{\tau(\bar{\chi})} \sum_{a_{1}=1}^{p-1} \cdots \sum_{a_{k}=1}^{p-1} \sum_{b=1}^{p-1} \bar{\chi}(b) e\left(\frac{b\left(a_{1}+\cdots+a_{k}+n \bar{a}_{1} \cdots \bar{a}_{k}\right)}{p}\right) \\
& =\frac{1}{\tau(\bar{\chi})} \sum_{a_{2}=1}^{p-1} \cdots \sum_{a_{k}=1}^{p-1} \sum_{a_{1}=1}^{p-1} \sum_{b=1}^{p-1} \bar{\chi}\left(b \bar{a}_{1}\right) e\left(\frac{b\left(1+\cdots+a_{k}\right)+n b \bar{a}_{1}^{k+1} \bar{a}_{2} \cdots \bar{a}_{k}}{p}\right) \\
& =\frac{1}{\tau(\bar{\chi})} \sum_{a_{2}=1}^{p-1} \cdots \sum_{a_{k}=1}^{p-1} \sum_{b=1}^{p-1} \bar{\chi}(b) e\left(\frac{b+b\left(a_{2}+\cdots+a_{k}\right)}{p}\right) \\
& \times \sum_{a_{1}=1}^{p-1} \chi\left(a_{1}\right) e\left(\frac{n b \bar{a}_{1}^{k+1} \bar{a}_{2} \cdots \bar{a}_{k}}{p}\right) \\
& =\frac{1}{\tau(\bar{\chi})} \sum_{a_{2}=1}^{p-1} \cdots \sum_{a_{k}=1}^{p-1} \sum_{b=1}^{p-1} \bar{\chi}(b) e\left(\frac{b+b\left(a_{2}+\cdots+a_{k}\right)}{p}\right) \\
& \times \sum_{a_{1}=1}^{p-1} \bar{\chi}^{r}\left(a_{1}\right) e\left(\frac{n b a_{1} \bar{a}_{2} \cdots \bar{a}_{k}}{p}\right) \\
& =\frac{\tau\left(\bar{\chi}^{r}\right)}{\tau(\bar{\chi})} \sum_{b=1}^{p-1} \bar{\chi}(b) e\left(\frac{b}{p}\right) \sum_{a_{2}=1}^{p-1} \cdots \sum_{a_{k}=1}^{p-1} \chi^{r}\left(n b \bar{a}_{2} \cdots \bar{a}_{k}\right) e\left(\frac{b\left(a_{2}+\cdots+a_{k}\right)}{p}\right) \\
& =\chi^{r}(n) \cdot \frac{\tau^{k+1}\left(\bar{\chi}^{r}\right)}{\tau(\bar{\chi})} \\
& =\chi^{r}\left(m c_{1} c_{2} \cdots c_{k}\right) \cdot \frac{\tau^{k+1}\left(\bar{\chi}^{r}\right)}{\tau(\bar{\chi})} \text {. }
\end{aligned}
$$

If $d>1$ and $\chi^{\frac{p-1}{d}} \neq \chi_{0}$, then from the method of proving (2.2), we have

$$
\sum_{a_{1}=1}^{p-1} \chi\left(a_{1}\right) e\left(\frac{n b \bar{a}_{1}^{k+1} \bar{a}_{2} \cdots \bar{a}_{k}}{p}\right)=0 .
$$


From this identity and the method of proving (3.1), we may immediately deduce that if $\chi^{\frac{p-1}{d}} \neq \chi_{0}$, then

$$
S\left(c_{1}, c_{2}, \ldots, c_{k}, m, \chi ; p\right)=0 .
$$

If $d>1$ and $\chi^{\frac{p-1}{d}}=\chi_{0}$, then $\chi$ must be a $d$ th character $\bmod p$, so there exists a character $\chi_{1} \bmod p$ such that $\chi=\chi_{1}^{d}$. Let $\chi_{d}$ be a $d$-order character $\bmod p$, then we have

$$
\begin{aligned}
& \sum_{a_{1}=1}^{p-1} \chi\left(a_{1}\right) e\left(\frac{n b \bar{a}_{1}^{k+1} \bar{a}_{2} \cdots \bar{a}_{k}}{p}\right) \\
& \quad=\sum_{a=1}^{p-1} \chi_{1}\left(a^{d}\right) e\left(\frac{n b \bar{a}^{d \cdot \frac{k+1}{d}} \bar{a}_{2} \cdots \bar{a}_{k}}{p}\right) \\
& \quad=\sum_{a=1}^{p-1} \bar{\chi}_{1}(a)\left(1+\chi_{d}(a)+\chi_{d}^{2}(a)+\cdots+\chi_{d}^{d-1}(a)\right) e\left(\frac{n b a^{\frac{k+1}{d}} \bar{a}_{2} \cdots \bar{a}_{k}}{p}\right) \\
& =\sum_{i=0}^{d-1} \sum_{a=1}^{p-1} \bar{\chi}_{1}(a) \chi_{d}^{i}(a) e\left(\frac{n b a^{\frac{k+1}{d}} \bar{a}_{2} \cdots \bar{a}_{k}}{p}\right) .
\end{aligned}
$$

Let $\left(\frac{k+1}{d}, p-1\right)=d_{1}$, then repeat the process of proving (2.1), (2.2) and (2.3). Combining (3.1), (3.2) and (3.3), we may immediately deduce the estimate

$$
\left|S\left(c_{1}, c_{2}, \ldots, c_{k}, m, \chi ; p\right)\right| \leq(k+1) \cdot p^{\frac{k}{2}}
$$

Now note that $|\tau(\chi)|=\left|\tau\left(\chi^{r}\right)\right|=\sqrt{p}$, Theorems 1 and 2 follow from (3.1), (3.2) and (3.4). Now we prove Theorem 3. If $q \geq 3$, we separate $q$ into two cases $(q, p-1)=1$ and $(q, p-1)=q$. If $(q, p-1)=q$, then note that for any $q$ th non-residue $r \bmod p$, we have

$$
\sum_{h=0}^{q-1} \chi_{q}^{l}\left(r^{h}\right)= \begin{cases}q & \text { if } q \mid l, \\ 0 & \text { if }(q, l)=1 .\end{cases}
$$

From (I) of Lemma 2, we can deduce that

$$
\begin{aligned}
& \sum_{i=0}^{q-1}\left[\sum_{a_{1}=1}^{p-1} \sum_{a_{2}=1}^{p-1} \cdots \sum_{a_{q-1}=1}^{p-1}\left(\frac{c_{1} a_{1}+c_{2} a_{2}+\cdots+c_{q-1} a_{q-1}+r^{i} \bar{a}_{1} \bar{a}_{2} \cdots \bar{a}_{q-1}}{p}\right)\right]^{2} \\
& \quad=p^{q-1} \sum_{i=0}^{q-1} \sum_{j=0}^{q-1} \sum_{h=0}^{q-1} \chi_{q}^{i+j}\left(c_{1} c_{2} \cdots c_{q-1} r^{h}\right) \frac{\tau^{q}\left(\chi_{2} \chi_{q}^{i}\right)}{\tau^{q}\left(\chi_{2}\right)} \cdot \frac{\tau^{q}\left(\chi_{2} \chi_{q}^{j}\right)}{\tau^{q}\left(\chi_{2}\right)} \\
& =q \cdot p^{q-1} \sum_{i=0}^{q-1} \sum_{j=0}^{q-1} \frac{\tau^{q}\left(\chi_{2} \chi_{q}^{i}\right)}{\tau^{q}\left(\chi_{2}\right)} \cdot \frac{\tau^{q}\left(\bar{\chi}_{2} \bar{\chi}_{q}^{i}\right)}{\tau^{q}\left(\chi_{2}\right)} \\
& =q^{2} \cdot p^{q-1} .
\end{aligned}
$$


If $(q, p-1)=1$, then from the method of proving (2.1) and the properties of Gauss sums, we can deduce that

$$
\begin{aligned}
& \sum_{i=0}^{q-1}\left[\sum_{a_{1}=1}^{p-1} \sum_{a_{2}=1}^{p-1} \cdots \sum_{a_{q-1}=1}^{p-1}\left(\frac{c_{1} a_{1}+c_{2} a_{2}+\cdots+c_{q-1} a_{q-1}+r^{i} \bar{a}_{1} \bar{a}_{2} \cdots \bar{a}_{q-1}}{p}\right)\right]^{2} \\
& \quad=\sum_{i=0}^{q-1} p^{q-1}=q \cdot p^{q-1} .
\end{aligned}
$$

If $q=2$ and $p \equiv 1 \bmod 4$, then applying (II) of Lemma 2, we have

$$
\begin{aligned}
\left(\sum_{a=1}^{p-1}\left(\frac{a+m \bar{a}}{p}\right)\right)^{2} & =p \cdot\left|1+\left(\frac{m}{p}\right) \cdot \frac{\tau^{2}\left(\bar{\chi}_{1}\right)}{\tau^{2}\left(\chi_{1}\right)}\right|^{2} \\
& =2 p+\left(\frac{m}{p}\right) \cdot \frac{\tau^{2}\left(\bar{\chi}_{1}\right)}{\tau^{2}\left(\chi_{1}\right)}+\left(\frac{m}{p}\right) \cdot \frac{\tau^{2}\left(\chi_{1}\right)}{\tau^{2}\left(\bar{\chi}_{1}\right)} .
\end{aligned}
$$

Therefore, from (3.5) we can deduce that

$$
\left(\sum_{a=1}^{p-1}\left(\frac{a+\bar{a}}{p}\right)\right)^{2}+\left(\sum_{a=1}^{p-1}\left(\frac{a+r \bar{a}}{p}\right)\right)^{2}=4 p .
$$

This proves Theorem 3.

To prove the corollary, note that

$$
\sum_{a=1}^{p-1}\left(\frac{a+m \bar{a}}{p}\right)=\sum_{a=1}^{\frac{p-1}{2}}\left(\frac{a+m \bar{a}}{p}\right)+\sum_{a=\frac{p+1}{2}}^{p-1}\left(\frac{a+m \bar{a}}{p}\right)=2 \sum_{a=1}^{\frac{p-1}{2}}\left(\frac{a+m \bar{a}}{p}\right) .
$$

From (3.5) we may immediately deduce the identity

$$
p=\left(\sum_{a=1}^{\frac{p-1}{2}}\left(\frac{a+r \bar{a}}{p}\right)\right)^{2}+\left(\sum_{b=1}^{\frac{p-1}{2}}\left(\frac{b+s \bar{b}}{p}\right)\right)^{2},
$$

where $r$ and $s$ are any two integers such that $\left(\frac{r}{p}\right) \cdot\left(\frac{s}{p}\right)=-1$.

This completes the proof of our corollary.

\section{Competing interests}

The authors declare that they have no competing interests.

\section{Authors' contributions}

$Y L$ carried out the upper bound estimate problem of one kind sums analogous to the high-dimensional Kloosterman sums. DH participated in the research and summary of the study.

\section{Author details}

${ }^{1}$ Department of Mathematics, Northwest University, Xi'an, Shaanxi, P.R. China. ${ }^{2}$ Department of Mathematics, Xi'an Shiyou University, Xi'an, Shaanxi, P.R. China.

\section{Acknowledgements}

The authors would like to thank the referee for carefully examining this paper and providing a number of important comments. This work is supported by the N.S.F. of P.R. China $(11071194,61202437)$, and by the Youth Science and Technology Innovation Foundation of Xi'an Shiyou University (2012QN012). 


\section{References}

1. Chowla, S: On Kloosterman's sum. Norske Vid. Selsk. Forh., Trondheim 40, 70-72 (1967)

2. Malyshev, AV: A generalization of Kloosterman sums and their estimates. Vestn. Leningr. Univ. 15, $59-75$ (1960) (in Russian)

3. Zhang, W: On the fourth power mean of the general Kloosterman sums. Indian J. Pure Appl. Math. 35, 237-242 (2004)

4. Cochrane, T, Zheng, Z: Bounds for certain exponential sums. Asian J. Math. 4, 757-774 (2000)

5. Cochrane, T, Zheng, Z: Upper bounds on a two-term exponential sums. Sci. China Ser. A 44, 1003-1015 (2001)

6. Cochrane, T, Zheng, Z: Pure and mixed exponential sums. Acta Arith. 91, 249-278 (1999)

7. Cochrane, T, Pinner, C: A further refinement of Mordell's bound on exponential sums. Acta Arith. 116, 35-41 (2005)

8. Cochrane, T, Pinner, C: Using Stepanov's method for exponential sums involving rational functions. J. Number Theory 116, 270-292 (2006)

9. Weil, A: On some exponential sums. Proc. Natl. Acad. Sci. USA 34, 204-207 (1948)

10. Burgess, DA: On Dirichlet characters of polynomials. Proc. Lond. Math. Soc. 13, 537-548 (1963)

11. Apostol, TM: Introduction to Analytic Number Theory. Springer, New York (1976)

12. Granville, A, Soundararajan, K: Large character sums: pretentious characters and the Pólya-Vinogradov theorem. J. Am. Math. Soc. 20, 357-384 (2007)

13. Zhang, W, Yao, W: A note on the Dirichlet characters of polynomials. Acta Arith. 115, 225-229 (2004)

14. Zhang, W, Yi, Y: On Dirichlet characters of polynomials. Bull. Lond. Math. Soc. 34, 469-473 (2002)

15. Zhang, W, Li, H: Elementary Number Theory. Shaanxi Normal University Press, Xi'an (2008)

doi:10.1186/1029-242X-2013-130

Cite this article as: Li and Han: A sum analogous to the high-dimensional Kloosterman sums and its upper bound estimate. Journal of Inequalities and Applications 2013 2013:130.

\section{Submit your manuscript to a SpringerOpen ${ }^{\ominus}$ journal and benefit from:}

- Convenient online submission

- Rigorous peer review

- Immediate publication on acceptance

- Open access: articles freely available online

- High visibility within the field

- Retaining the copyright to your article 